TAMKANG JOURNAL OF MATHEMATICS

Volume 33, Number 4, Winter 2002

\title{
MONOTONE ITERATIVE TECHNIQUE FOR CARATHEODORY THEORY OF NONLINEAR FUNCTIONAL RANDOM INTEGRAL EQUATIONS
}

\author{
B. C. DHAGE
}

\begin{abstract}
In this paper some random fixed point theorems for the mappings on an ordered Banach space are proved. As applications, the existence of the extremal solutions of some nonlinear functional random integral equations is obtained under certain monotonicity conditions.
\end{abstract}

\section{Introduction}

Let $(\Omega, \mathcal{A})$ be a measurable space and set $X$ be a real separable Banach space. Let $\beta_{X}$ denote the $\sigma$-algebra of all Borel subsets of $X$. A mapping $x: \Omega \rightarrow X$ is called measurable if for any $B \in \beta_{X}, x^{-l}(B)=\{\omega \in \Omega: x(\omega) \in B\} \in \mathcal{A}$. A mapping $T: \Omega^{\prime} \times X \rightarrow X$ is called a random operator if $T(\cdot, x)$ is measurable for all $x \in X$. A random operagor $T$ is also denoted by $T(\omega) x:=T(\omega, x)$ or by simply $T(\omega)$. A random operator $T(\omega)$ is called continuous if $T(\omega)(\cdot)$ is continuous for each $\omega \in \Omega$, and completely continuous, if it is continuous and for any bounded set $B$ in $X, T(\omega)(B)$ is precompact subset of $X$ for each $\omega \in \Omega$. Similarly a random operator $T: \Omega \times X \rightarrow X$ is called contraction if $\|T(\omega) x-T(\omega) y\| \leq \alpha(\omega)\|x-y\|(\alpha(\omega)<l)$ for all $x, y \in X$ and for each $\omega \in \Omega$. A measurable mapping $\xi: \Omega \rightarrow X$ is called a random fixed point of the random operator $T(\omega)$ if $T(\omega) \xi(\omega)=\xi(\omega)$ for each $\omega \in \Omega$.

A non-empty closed subset $K$ of $X$ is called a cone in $X$ if (i) $K+K \subseteq K$ (ii) $\lambda K \subseteq K$ for $\lambda \in \mathbf{R}, \lambda \geq 0$ and (iii) $\{-K\} \cap K=0$, where 0 is a zero emelment of $X$. Now we introduce an order relation $\leq$ in $X$ as follows : Let $x, y \in X$. Then $x \leq y$ if and only if $y-x \in K$. The Banach spcae $X$ together with this order relation $\leq$ becomes a ordered Banach space and it is denoted by $(\mathrm{X},\|\cdot\|, \leq)$ or simply by $(X, K)$. We need the following properties of the cone $K$ in the sequel. A cone $K$ in $X$ is called normal if every order bounded set in $X$ is bounded in norm. Similaryly a cone $K$ in $X$ is called regular if every increasing sequence which is bounded above is convergent. Let $u, v \in X$ be such that $u \leq v$. Then an order interval $[u, v]$ is a set in $X$ defined by $[u, v]=\{x \in X \mid u \leq x \leq v\}$. A random operator $T(w)$ is called nondecreasing if for any $x, y \in X, x \leq y$ implies $T(\omega) x \leq T(\omega) y$ for each $\omega \in \Omega$. A measurable mapping

Received October 15, 2001; revised March 12, 2002.

2000 Mathematics Subject Classification. 47H10

Key words and phrases. Fixed point theorem, random integral equation. 
$\xi^{*}: \Omega \rightarrow X$ is called a maximal random fixed point of the random operator $T(\omega)$ if for any random fixed point $\xi$ of $T(\omega), \xi(\omega) \leq \xi^{*}(w)$ for each $\omega \in \Omega$. Similarly a minimal random fixed point $\xi_{*}$ of the random operator $T(\omega)$ is defined.

Several classical or deterministic fixed point theorems have been extended to random operators in the literature by different authors and some of the random fixed point theorems have nice applications to nonlinear random differenital and integral equations for proving the existence and uniqueness results. See for details, Bharucha Reid [3] and the references given therein. In this paper we prove some fixed point theorems involving a single and a pair of random operators on an ordered Banach space which extend the classical or deterministic well-known fixed point theorems of Amann [1] and Dhage [4] to random operators.

\section{Random Fixed Point Theory}

Before going to the mian results of this paper, we recall some useful definitions:

The measure of noncompactness of a bounded set $S$ in a Banach space $X$ is a nonnegative real number $\alpha(S)$ to be defined by

$$
\alpha(S)=\inf \left\{r>0 ; S=\bigcup_{i=l}^{n} S_{i} \operatorname{diam}\left(S_{i}\right) \leq r, \forall_{i}\right\}
$$

The function $\alpha(\cdot)$ is called a Kuratowskii measure of noncompactness and the details may be found in Banas and Goebel [2]. A random operator $T: \Omega \times X \rightarrow X$ is called $\alpha$-condensing if for any bounded set $S$ in $X, T(\omega)(S)$ is bounded and $\alpha(T(\omega) S)<\alpha(S)$ if $\alpha(S)>0$ for each $\omega \in \Omega$. It is know that every completely continuous and contraction random operators are $\alpha$-condensing.

Let $a, b: \Omega \rightarrow X$. Then by $a \leq b$ we mean $a(\omega) \leq b(\omega) \forall \omega \in \Omega$. In this case we also write $a \leq b$ on $\Omega$. By the order interval $[a, b]$ we denote a set in $X$ defined by

$$
\begin{aligned}
{[a, b] } & =\{x \in X \mid a(\omega) \leq x \leq b(\omega) \quad \forall \omega \in \Omega\} \\
& =\cap_{\omega \in \Omega}[a(\omega), b(\omega)] .
\end{aligned}
$$

Our first result is

Theorem 2.1. Let $a, b: \Omega \rightarrow X$ be two measurable functions such that $a \leq b$ and let $Q: \Omega \times[a, b] \rightarrow[a, b]$ be a continuous and nondecreasing random operator. Then $Q$ has a minimal random fixed point $\xi *$ and a maximal random fixed point $\xi^{*}$ if any one of following conditions holds.

(a) $Q(\omega)$ is $\alpha$-condensing and the cone $K$ in $X$ is normal.

(b) The cone $K$ is regular in $X$.

Moreover,

$$
\xi_{*}(\omega)=\lim _{n} Q^{n}(\omega) a \text { and } \xi^{*}(\omega)=\lim _{n} Q^{n}(\omega) b
$$


Proof. Let $\omega \in \Omega$ be a fixed element. Then the sequences $\left\{x_{n}(\omega)\right\}$ and $\left\{y_{n}(\omega)\right\}$ in $[a, b]$ defined by

$$
x_{n}(\omega)=Q^{n}(\omega) a \text { and } y_{n}(\omega)=Q^{n}(\omega) b
$$

satisfy the inequality

$$
a(w)=x_{0}(\omega) \leq x_{l}(\omega) \leq \cdots \leq x_{n}(\omega) \leq \cdots \leq y_{n}(\omega) \leq y_{0}(\omega)=b(\omega) .
$$

for each $\omega \in \Omega$.

Since $a$ and $b$ are measurable function on $\Omega$, and $Q(\omega)$ is a continuous random operator, each $x_{n}(\omega)$ and $y_{n}(\omega)$ are measurable and consequently are random variables for each $n \in \mathbf{N}$. We discuss the conclusion of the theorem in the following two cases:

Case I. Suppose that the cone $K$ in $X$ is normal. Then the order interval $[a, b]$ is a bounded subset of $X$. Consequently $\left\{x_{n}(\omega)\right\}$ and $\left\{y_{n}(\omega)\right\}$ are bounded sets in $X$. We show that $\left\{x_{n}(\omega)\right\}$ and $\left\{y_{n}(\omega)\right\}$ are precompact sets in $X$. Suppose that $\left\{x_{n}(\omega)\right\}$ is not precompact.

Now

$$
\left\{x_{n}(\omega)\right\}=\{a(\omega)\} \cup Q\left(\left\{x_{n}(\omega)\right\}\right) .
$$

Hence

$$
a\left(\left\{x_{n}(\omega)\right\}\right) \leq \alpha\left(Q(\omega)\left(\left\{x_{n}\right\}\right)\right)<\alpha\left(x_{n}(\omega)\right) \text { which is a contradiction. }
$$

Hence $\left\{x_{n}(\omega)\right\}$ is precompact and $\left\{\overline{x_{n}(\omega)}\right\}$ is compact for each $\omega \in \Omega$. Therefore there is a point $\xi_{*}$ in $[a, b]$ such that $\lim _{n} x_{n}(\omega)=\xi_{*}(\omega)$. Similarly it is proved that there is a point $\xi^{*}$ in $[a, b]$ such that $\lim _{n} y_{n}(\omega)=\xi^{*}(\omega)$. The measurability of $\left\{x_{n}(\omega)\right\}$ and $\left\{y_{n}(\omega)\right\}$ implies that $\xi_{*}$ and $\xi^{*}$ are random variables. From the continuity of $Q(\omega)$ it follows that $Q(\omega) \xi_{*}(\omega)=\xi_{*}(\omega)$ and $Q(\omega) \xi^{*}(\omega)=\xi^{*}(\omega)$ for each $\omega \in \Omega$. Now suppose that $\xi$ is any other random fixed point of $Q(\omega)$ in $[a, b]$. Then $a(\omega) \leq \xi(\omega) \leq b(\omega)$ for each $\omega \in \Omega$ and from the nondecreasing nature of $Q(\omega)$ it follows that

$$
x_{n}(\omega)=Q^{n}(\omega) a \leq \xi(\omega) \leq Q^{n}(\omega) b=y_{n}(\omega) \text { for each } \omega \in \Omega .
$$

Hence $\xi_{*}(\omega) \leq \xi(\omega) \leq \xi^{*}(\omega)$ for each $\omega \in \Omega$. Thus $\xi_{*}$ and $\xi^{*}$ are respectively the minimal and maximal random fixed points of $Q(\omega)$ in $[a, b]$.

Case II. Next suppose that the condition (b) holds. By the regularity of cone $K$ in $X$, there are points $\xi_{*}$ and $\xi^{*}$ in $[a, b]$ such that $\ln _{n} x_{n}(\omega)=\xi_{*}(\omega)$ and $\lim _{n} y_{n}(\omega)=$ $\xi^{*}(\omega), \omega \in \Omega$, where $x_{n}(\omega)$ and $y_{n}(\omega)$ are defined by (2.3). The rest of the proof is similar to Case I and we omit the details. This completes the proof.

Corollary 2.1. Let $a, b: \Omega \rightarrow X$ be two measurable functions such that $a \leq b$ and let $Q: \Omega \times[a, b] \rightarrow[a, b]$ be a completely continuous and nondecreasing random operator. 
Further if the cone $K$ in $X$ is normal, then $Q(\omega)$ has a minimal random fixed point $\xi_{*}$ and a maximal random fixed point $\xi^{*}$ in $[a, b]$. Moreover,

$$
\xi_{*}(\omega)=\lim _{n} Q^{n}(\omega) a \quad \text { and } \quad \xi^{*}(\omega)=\lim _{n} Q^{n}(\omega) b
$$

Theorem 2.2. Let $Q: \Omega \times X \rightarrow X$ be a continuous and nondecreasing random operator. Suppose that there exist two measurable functions $a, b: \Omega \rightarrow X$ such that $a \leq b$ satisfying $a(\omega) \leq Q(\omega) a$ and $Q(\omega) b \leq b(\omega)$ for each $\omega \in \Omega$. Then $Q(\omega)$ has a minimal random fixed point $\xi_{*}$ and a maximal random fixed point $\xi_{*}$ in $[a, b]$ if any one of the following conditions holds.

(a) $Q(\omega)$ is $\alpha$-condensing and the cone $K$ in $X$ is normal.

(b) The cone $K$ in $X$ is regular.

Moreover

$$
\xi_{*}(\omega)=\lim _{n} Q^{n}(\omega) a \quad \text { and } \quad \xi^{*}(\omega)=\lim _{n} Q^{n}(\omega) b
$$

Proof. Consider the order interval $[a, b]$ in $X$. We shall show that $Q$ defines a mapping $Q(\omega): \Omega \times[a, b] \rightarrow[a, b]$. Let $x \in[a, b]$ be any element. Then $a \leq x \leq b$. Since $Q$ is nondecreasing and $a(\omega) \leq Q^{n}(\omega) a$ and $Q^{n}(\omega) b \leq b(\omega)$ for each $\omega \in \Omega$, one has

$$
a(\omega) \leq Q(\omega) a \leq Q(\omega) x \leq Q(\omega) b \leq b(\omega)
$$

for each $\omega \in \Omega$. Hence $Q(\omega) x \in[a, b]$ for all $x \in[a, b]$. Now the desired conclusion follows by an application of Theorem 2.1.

Corollary 2.2. Let $Q: \Omega \times X \rightarrow X$ be a completely continuous and nondecreasing random operator. Suppose that there exist two measurable functions $a, b: \Omega \rightarrow X$ such that $a \leq b$ satisfying $a(\omega) \leq Q(\omega) a$ and $Q(\omega) b \leq b(\omega)$ for each $\omega \in \Omega$. Further if the cone $K$ in $X$ is normal, then the operator $Q(\omega)$ has a minimal random fixed point $\xi_{*}$ and a maximal random fixed point $\xi^{*}$ in $[a, b]$. Moreover,

$$
\xi_{*}(\omega)=\lim _{n} Q^{n}(\omega) a \text { and } \xi^{*}(\omega)=\lim _{n} Q^{n}(\omega) b \text { for each } \omega \in \Omega .
$$

Theorem 3.3. Let $a, b: \Omega \rightarrow X$ be two measurable functions such that $a \leq b$ and let $A, B: \Omega \times[a, b] \rightarrow[a, b]$ be two non-decreasing random operators such that for each $\omega \in \Omega$,

(a) $A(\omega)$ is contraction,

(b) $B(\omega)$ is completely continuous, and

(c) $A(\omega) x+B(\omega) x \in[a, b]$ for each $x \in[a, b]$.

Further if the cone $K$ in $X$ is normal, then the operator equation

$$
A(\omega) x+B(\omega) x=x
$$


has a minimal random solution $\xi_{*}$ and a maximal random solution $\xi^{*}$ in $[a, b]$. Moreover,

$$
\left.\begin{array}{c}
\xi_{*}(\omega)=\lim _{n} x_{n}(\omega) \text { and } \xi^{*}(\omega)=\lim _{n} y_{n}(\omega), \\
\text { where } \quad x_{n+1}(\omega)=A(\omega) x_{n}+B(\omega) x_{n}, \quad n \geq 0, \quad \text { with } x_{0}=a \\
\text { and } \quad y_{n+1}(\omega)=A(\omega) y_{n}+B(\omega) y_{n}, \quad n \geq 0, \quad \text { with } y_{0}=b
\end{array}\right\}
$$

Proof. Define a mapping $Q: \Omega \times X \rightarrow X$ by

$$
Q(\omega) x=A(\omega) x+B(\omega) x
$$

for each $\omega \in \Omega$. Clearly by hypothesis $(c), Q$ defines a mapping $Q: \Omega \times[a, b] \rightarrow[a, b]$. Also $Q$ is nondecreasing random operator in view of the fact that the sum of two measurable functions is again measurable. As $A(\omega)$ is contraction, it is continuous and since sum of two continuous mappings is again continuous, the random operator $Q(\omega)$ is continuous. It is easily shown that $Q$ is a $\alpha$-condensing random ooperator on $\Omega \times[a, b]$. Now $Q(\omega)$ satisfies all the conditions of Theorem 2.1 and hence a direct application of it yields the desired conclusion. The proof is complete.

Corollary 3.3. Let $A, B: \Omega \times X \rightarrow X$ be two non-decreasing random operators such that

(a) $A(\omega)$ is a contraction,

(b) $B(\omega)$ is completely continuous, and

(c) there exist two measurable functions $a, b: \Omega \rightarrow X$ such that $a \leq b$ and satisfy for each $\omega \in \Omega$,

$$
a(\omega) \leq A(\omega) a+B(\omega) a \text { and } A(\omega) b+B(\omega) b \leq b(\omega) .
$$

Further if the cone $K$ in $X$ is normal, then the operator equation (2.4) has a minimal random solution $\xi_{*}$ and a miximal random solution $\xi^{*}$ in $[a, b]$. Moreover,

$$
\xi_{*}(\omega)=\lim _{n} x_{n}(\omega) \text { and } \xi^{*}(\omega)=\lim _{n} y_{n}(\omega), \text { for each } \omega \in \Omega,
$$

where $\left\{x_{n}(\omega)\right\}$ and $\left\{y_{n}(\omega)\right\}$ are defined by $(2.5)$.

To state our final fixed point theorem we need the following porperty of the cone $K$ in $X$.

A cone $K$ in a seperable Banach algebra $X$ is called a positive if it satisfies $(d) K_{0} K \subseteq K$ where "o" is a multiplicative composition in $X$.

A random operator $T: \Omega \times X \rightarrow X$ is called positive if range $(T) \subset K \mid\{0\}$.

Lemma 2.1. Let $K$ be a positive cone $K$ in a Banach algebra $X$ and let $u_{1}, u_{2}, v_{1}$, $v_{2} \in K$. If $u_{1} \leq u_{2}$ and $v_{1} \leq v_{2}$, then $u_{1} v_{1} \leq u_{2} v_{2}$.

Proof.

$$
\text { Now } \begin{aligned}
u_{2} v_{2}-u_{1} v_{1}- & u_{2} v_{2}-u_{1} v_{2}+u_{1} v_{2}-u_{1} v_{1} \\
& =\left(u_{2}-u_{1}\right) v_{2}+u_{1}\left(v_{2}-v_{1}\right) \\
& =k_{1} v_{2}+u_{1} k_{2} \\
& =k
\end{aligned}
$$


for some $k=k_{1} v_{2}+u_{1} k_{2} \in K$. By the definition of the order relation $\leq$ which implies that $u_{1} v_{1} \leq u_{2} v_{2}$.

Theorem 3.4. Let $a, b: \Omega \rightarrow K$ be two measurable functions such that $a \leq b$ and let $A, B: \Omega \times[a, b] \rightarrow X$ be two positive and non-decreasing random operators satisfying for each $\omega \in \Omega$.

(a) $A(\omega)$ is Lipschitziian with Lipschitz constant $\alpha(\omega)$,

(b) $B(\omega)$ is completely continuous, and

(c) $A(\omega) x B(\omega) x \in[a, b]$ for each $x \in[a, b]$.

Further if the cone Kin $X$ is positive and normal, then the random equation

$$
A(\omega) x B(\omega) x=x
$$

has a minimal random solution $\xi_{*}$ and a maximal positive random solution $\xi^{*}$ in $[a, b]$ whenever $\alpha(\omega) M(\omega)<l$ for each $\omega \in \Omega$, where $M(\omega)=\|B(\omega)([a, b])\|=\sup \{\|B(\omega) x\|$ : $x \in[a, b]\}$.

Moreover,

$$
\xi_{*}(\omega)=\lim _{n} x_{n}(\omega) \text { and } \xi^{*}(\omega)=\lim _{n} y_{n}(\omega),
$$

$\left.\begin{array}{ll}\text { where } & x_{n+1}(\omega)=A(\omega) x_{n} B(\omega) x_{n}, \quad n \geq 0, \quad \text { with } x_{0}=a \\ \text { and } & y_{n+1}(\omega)=A(\omega) y_{n} B(\omega) y_{n}, \quad n \geq 0, \quad \text { with } y_{0}=b\end{array}\right\}$

Proof. Define a mapping $Q: \Omega \times X \rightarrow X$ by

$$
Q(\omega) x=A(\omega) x B(\omega) x .
$$

Now $A(\omega)$ and $B(\omega)$ are continuous and so from the continuity of multiplication in Banach algebra, it follows that $Q$ is a random operator. By hypothesis $(c), Q$ defines a mapping $Q: \Omega \times[a, b] \rightarrow[a, b]$. It is shown as in Dhange [4] that $Q(\omega)$ is a continuous and $\alpha$-condenring random operator. Also $Q(\omega)$ is nondecreasing is view of Lemma 2.1. Now the desired result follows by an application of Theorem 2.1.

Corollary 3.4. Let $A, B: \Omega \times X \rightarrow X$ be two non decreasing and positive random operators satisfying for each $\omega \in \Omega$.

(a) $A(\omega)$ is Lipschitzian with Lipschitz constant $\alpha(\omega)$,

(b) $B(\omega)$ is completely continuous, and

(c) there exist measurable functions $a, b: \Omega \rightarrow K \mid\{0\}$ such that $a(\omega) \leq A(\omega) a B(\omega) a$ and $A(\omega) b B(\omega) b \leq b(\omega)$.

Further if the cone $K$ in $X$ is positive and normal, then the operator equation (2.7) has a minimal random fixed point $\xi_{*}$ and a maximal fixed point $\xi^{*}$ in $[a, b]$ whenever $a(\omega) M(\omega)<l$ for each $\omega \in \Omega$, where $M(\omega)=\|B(\omega)([a, b])\|$ Moreover,

$$
\begin{aligned}
& \xi_{*}(\omega)=\lim _{n} x_{n}(\omega), \quad \text { and } \\
& \xi^{*}(\omega)=\lim _{n} y_{n}(\omega)
\end{aligned}
$$


where $\left\{x_{n}(\omega)\right\}$ and $\left\{y_{n}(\omega)\right\}$ are defined by $(2.8)$.

In the following section we shall obtain the existence of extremal solutions of a certain nonlinear functional random integral equations.

\section{Functional Random Integral Equations}

Given a closed and bounded interval $J=[0,1]$ in $\boldsymbol{R}$, the set of all real numbers, consider the nonlinear functional random integral equation (in short RIE).

$$
x(t, \omega)=q(t, \omega)+\int_{0}^{\sigma(t)} f(s, x(\eta(s), \omega), \omega) d s
$$

for all $t \in J$, here $q: J \times \Omega \rightarrow \boldsymbol{R}, f: J \times \boldsymbol{R} \times \Omega \rightarrow \boldsymbol{R}$ and $\sigma, \eta: J \rightarrow J$. The special cases of the RIE(3.1) have been studied extensively in the literature. See Bharucha - Reid [3].

We shall obtain the existence of the extremal random solutions of the $\operatorname{RIE}(3.1)$ in the space $B M(J, \boldsymbol{R})$ of all measurable and bounded real-valued functions defined on $J$. We equip the space $B M(J, \boldsymbol{R})$ with a norm $\|\cdot\|$ defined by

$$
\|x\|=\sup _{t \in J}\|x(t)\| .
$$

Clearly $B M(J, \boldsymbol{R})$ is a separable Banach space with this supremum norm. We define an order relation $\leq$ in the space $B M(J, \boldsymbol{R})$ by the cone $K$ in $B M(J, \boldsymbol{R})$ define by

$$
K=\{x \in B M(J, \boldsymbol{R}) \mid x(t) \geq 0, \quad t \in J\}
$$

Clerly the cone $K$ is normal $B M(J, \boldsymbol{R})$.

A function $u: \Omega \rightarrow B M(J, \boldsymbol{R})$ is called a lower solution of the $\mathrm{RIE}(3.1)$ if for each $\omega \in \Omega$,

$$
u(t, \omega) \leq q(t, \omega)+\int_{0}^{\sigma(t)} f(s, x(\eta(s), \omega), \omega) d s
$$

for all $t \in J$, Simillarly $u$ is called an upper solution of the RIE (3.1) if the reverse inequality is satisfied. Finally a function $u: \Omega \rightarrow B M(J, \boldsymbol{R})$ is called a solution of the RIE (3.1) if it is a lower as well as an upper solution of the RIE (3.1) on $J$. In this case $u$ is called a wide sense solution of RIE (3.1). Moreover if $u(t, \omega)$ is mesarable in $\omega$, then it is called a random solution of RIE (3.1).

We need the following definition in the sequel.

Definition 3.1. A function $\beta: J \times \boldsymbol{R} \times \Omega \rightarrow \boldsymbol{R}$ is said to be $L_{\omega}^{1}$-Caratheodory, if for each $\omega \in \Omega$,

(i) $t \rightarrow \beta(t, x, \omega)$ is measurable for all $x \in \boldsymbol{R}$,

(ii) $x \rightarrow \beta(t, \cdot, \omega)$ is continuous for almost all $t \in J$, and

(iii) for given $k>0$, there exists a function $h_{k}: \Omega \rightarrow L^{1}(J, \boldsymbol{R})$ such that 


$$
|\beta(t, x, \omega)| \leq h_{k}(t, \omega), \quad \text { a,e } t \in J .
$$

for all $x \in \boldsymbol{R}$ with $|x| \leq k$.

We consider the following set of asscumptions

$\left(\mathbf{H}_{\mathbf{0}}\right)$ The functions $\sigma, \eta: J \rightarrow J$, are continuous.

$\left(\mathbf{H}_{\mathbf{1}}\right) t \rightarrow q(t, \omega)$ is continuous for each $\omega \in \Omega$ and $\omega \rightarrow q(t, \omega)$ is measurable for all $t \in J$

$\left(\mathbf{H}_{\mathbf{2}}\right)$ The function $\omega \rightarrow f(t, x, \omega)$ is measurable for all $(t, x) \in J \times \boldsymbol{R}$.

$\left(\mathbf{H}_{3}\right) f(t, x, \omega)$ is $L_{\omega}^{1}$-Caratheodory.

$\left(\mathbf{H}_{4}\right)$ For each $\omega \in \Omega, f(t, x, \omega)$ is nondecreasing in $x$ for almost all $t \in J$.

$\left(\mathbf{H}_{\mathbf{5}}\right)$ There exists two measurable functions $a, b: \Omega \rightarrow B M(J, \boldsymbol{R})$ such that $a \leq b$ and $a$ and $b$ are respectively the lower and upper solutions of the RIE (3.1) on $J$.

Theorem 3.1. Assume that the hypotheses $\left(\mathbf{H}_{\mathbf{0}}\right)-\left(\mathbf{H}_{\mathbf{5}}\right)$ hold. Then the RIE (3.1) admits a maximal random solution $\xi^{*}$ and a minimal random solution $\xi_{*}$ in $[a, b]$.

Moreover,

$$
\xi_{*}(\omega)=\lim _{n} x_{n}(\omega) \quad \text { and } \quad \xi^{*}(\omega)=\lim _{n} y_{n}(\omega)
$$

where

$$
x_{n}(\omega)=q(t, \omega)+\int_{0}^{\sigma(t)} f\left(s, x_{n-1}(\eta(s), \omega), \omega\right) d s, \quad n \geq 1, \quad x_{0}(\omega)=a(\omega),
$$

and

$$
y_{n}(\omega)=q(t, \omega)+\int_{0}^{\sigma(t)} f\left(s, y_{n-1}(\eta(s), \omega), \omega\right) d s, \quad n \geq 1, \quad y_{0}(\omega)=b(\omega)
$$

Proof. Define a mapping $Q$ on $\Omega \times B M(J, \boldsymbol{R})$ by

$$
Q(\omega) x(t)=q(t, \omega)+\int_{0}^{\sigma(t)} f(s, x(\eta(s), \omega), \omega) d s, \quad t \in J
$$

Take

$$
y(t, \omega)=\int_{0}^{\sigma(t)} f(s, x(\eta(s), \omega), \omega) d s, \quad t \in J \text { and } \omega \in \Omega
$$

Since $\sigma$ is continuous, $t \rightarrow y(t, \omega)$ is continuous and hence is bounded on $J$ for each $\omega \in \Omega$

Further $\omega \rightarrow y(t, \omega)=\int_{0}^{\sigma(t)} f(s, x(\eta(s), \omega), \omega) d s$ exists for each $\omega \in \Omega$, and hence is a limit of the finite sum of the measurable functions. So $y: \Omega \rightarrow B M(J, \boldsymbol{R})$ is a measurable function. This further in view of the hypothysis $\left(\mathbf{H}_{\mathbf{1}}\right)$ imples that $Q$ defines a measurable mapping $Q: \Omega \times B M(J, \boldsymbol{R}) \rightarrow B M(J, \boldsymbol{R})$ and hence $Q$ is a random operator. By hypothesis $\left(\mathbf{H}_{\mathbf{5}}\right), a(\omega) \leq Q(\omega) a$ and $Q(\omega) b \leq b(\omega)$ for each $\omega \in \Omega$. 
Let $x, y \in B M(J, \boldsymbol{R})$ be such that $x \leq y$. Then by $\left(\mathbf{H}_{4}\right)$ for each $\omega \in \Omega$,

$$
\begin{aligned}
Q(\omega) x(t) & =q(t, \omega)+\int_{0}^{\sigma(t)} f(s, x(\eta(s), \omega), \omega) d s \\
& \leq q(t, \omega)+\int_{0}^{\sigma(t)} f(s, x(\eta(s), \omega), \omega) d s \\
& =Q(\omega) y(t)
\end{aligned}
$$

for all $t \in J$, and so $Q(\omega) x \leq Q(\omega) y$ for each $\omega \in \Omega$. Hence $Q(\omega)$ is a nondecreasing random operator on $B M(J, \boldsymbol{R})$. Next we show that $Q(\omega)$ is a completely continuous random operator on $B M(J, \boldsymbol{R})$. Obviously by using a standard arguments (see Granas et.at.[5]) and using dominated convergence theorem, it is shown that $Q(\omega)$ is a continuous random operator. Let $k>0$ be a fixed real number and consider a sequence $\left\{x_{n}\right\}$ in $B M(J, \boldsymbol{R})$ such that $\left|x_{n}(t)\right| \leq k$ for all $t \in J$. Then by $\left(\mathbf{H}_{\mathbf{8}}\right)$,

$$
\begin{aligned}
\left|Q(\omega) x_{n}(t)\right| & \leq\left|\int_{0}^{\sigma(t)} f(s, x(\eta(s), \omega), \omega) d s\right|+|q(t, \omega)| \\
& \leq\|q(\omega)\|+\int_{0}^{\sigma(t)} h_{k}(s, \omega) d s \\
& \leq\|q(\omega)\|+\left\|h_{k}(\omega)\right\|_{L^{1}}
\end{aligned}
$$

which shows that $\left\|Q(\omega) x_{n}\right\|$ is uniformly bounded for each $\omega \in \Omega$. Now let $t, \tau \in J$. Then

$$
\left|Q(\omega) x_{n}(t)-Q(\omega) x_{n}(\tau)\right| \leq|q(t, \omega)-q(\tau, \omega)|+|p(t, \omega)-p(\tau, \omega)|
$$

where $p(t, \omega)=\int_{0}^{\sigma(t)} h_{k}(s, \omega) d s$.

Since $q(\cdot, \omega)$ and $p(\cdot, \omega)$ are uniformly continuous on $J$, it follows that $\left\{Q(\omega) x_{n}(t)\right\}$ is a equi-continous set in $B M(J, \boldsymbol{R})$. Hence $Q(\omega)$ is a completely continuous random operator on $M(J, \boldsymbol{R})$ by Arzela-Ascoli theorem. Now the desired conclusion follows by application of Corollary 2.2. This completes the proof.

As an application of the main result of this section, we consider the functional first order random differential equation (in short RDE)

$$
\left.\begin{array}{ll}
\frac{d x(t, \omega)}{d t} & =f(t, x(\eta(t), \omega), \omega) \quad \text { a.e. } \quad t \in J . \\
x(0, \omega) & =q(\omega) \in \boldsymbol{R}
\end{array}\right\}
$$

for each $\omega \in \Omega$, here $f: J \times \boldsymbol{R} \times \Omega \rightarrow \boldsymbol{R}$ and $\eta: J \rightarrow J$ is continuous.

By the random solution of the $\operatorname{RDE}(3.6)$ we mean a measurable function $\xi: \Omega \rightarrow$ $A C(J, \boldsymbol{R})$ which satisfies $(3.6)$ on $J$.

We equip the space $A C(J, \boldsymbol{R})$ with a norm $\|\cdot\|$ and order relation $\leq$ by

$$
\|x\|=\sup _{t \in J}|x(t)|,
$$


and $x \leq y \Leftrightarrow x(t) \leq y(t)$ for all $t \in J$. by

Clearly $A C(J, \boldsymbol{R})$ is a ordered separable Banach space, where in the cone $K$ defined

$$
K=\{x \in A C(J, \boldsymbol{R}) \mid x \geq 0\}
$$

is normal.

We need the following set of hypothyses:

$\left(\mathbf{A}_{\mathbf{1}}\right) q: \Omega \rightarrow \boldsymbol{R}$ is measurable.

$\left(\mathbf{A}_{2}\right)$ There exists two measurable functions $u, v: \Omega \rightarrow A C(J, \boldsymbol{R})$ such that $u \leq v$, and for each $\omega \in \Omega$,

$$
\begin{aligned}
\frac{d u(t, \omega)}{d t} & \leq f(t, u(\eta(t), \omega), \omega) \quad \text { a.e. } t \in J . \\
u(0, \omega) & \leq q(\omega) \\
\text { and } \quad \frac{d v(t, \omega)}{d t} & \geq f(t, v(\eta(t), \omega), \omega) \quad \text { a.e. } t \in J . \\
v(0, \omega) & \leq q(\omega) .
\end{aligned}
$$

Theorem 3.2. Assume that the hypotheses $\left(\mathbf{A}_{1}\right)-\left(\mathbf{A}_{\mathbf{2}}\right)$ and $\left(\mathbf{H}_{2}\right)-\left(\mathbf{H}_{\mathbf{4}}\right)$ hold. Then the $\mathrm{RDE}(3.6)$ admits a minimal random solution $\xi_{*}$ and a maximal random solution $\xi^{*}$ in $[u, v]$. Moreover

$$
\xi_{*}(\omega)=\lim _{n} x_{n}(\omega) \text { and } \xi^{*}(\omega)=\lim _{n} y_{n}(\omega)
$$

for each $\omega \in \Omega$, where

with

$$
x_{n}(\omega)=q(\omega)+\int_{0}^{t} f\left(s, x_{n-1}(\eta(s), \omega), \omega\right) d s, \quad n \geq 1, \quad \text { with } \quad x_{0}=u .
$$

$$
y_{n}(\omega)=q(\omega)+\int_{0}^{t} f\left(s, x_{n-1}(\eta(s), \omega), \omega\right) d s, \quad n \geq 1, \quad \text { with } \quad y_{0}=v
$$

Proof. The problem (2.6) is equivalent to the RIE

$$
x(t, \omega)=q(\omega)+\int_{0}^{t} f(s, x(\eta(s), \omega), \omega) d s, \quad t \in J .
$$

Now the desired conclusion follows by and application of Theorem 3.1 since $A C(J, \boldsymbol{R}) \subset B M(J, \boldsymbol{R})$.

\section{Conclusion}

Finally while concluding this paper, we mention that we have not been able to relax the continuity of the random mappings in any result of this paper for proving the fixed point theorem or for proving the existence results for random differential and integral 
equations. This is because of the reason that the image of a random variable under a discontinuous random operator may not be a random variable. Again the fixed point theorems of this paper have some nice applications to a variety of nonlinear random differential and integral equations and some of the results in this direction will be reported else where.

\section{References}

[1] H. Amann, Fixed point equations and nonlinear eigenvalue problems in ordered Banach space, SIAM Review 18(1978), 620-709.

[2] J. Banas and K. Goebel, Measures of Noncompactness in Banach Spaces, Marcel Dekker, Inc - New York, 1980.

[3] A. T. Bharucha Reid, Random Integral Equations, Academic Press 1972.

[4] B. C. Dhage, On $\alpha$ condensing mappings in Banach algebras, The Math Student 63(1994), 146-152.

[5] A. Gramas, R. B. Gueuther and J. W. Lee, Some general existence principles in the Caratheodory theory of nonlinear differential equations, J. Math. pures et Appl. 70(1991), 153-196.

[6] S. Heikkila and V. Lakshmikanthan, Monotone Iterative Technique for Discontinuous Nonlinear Differential Equations, Marcel Dekker Inc. New York, 1994.

[7] S. Itoh, Random fixed point theorems with applicatioins to random differential equations in Banach space, J. Math. Anal. appl. 67(1979), 261-273.

[8] M. A. Krasonoselskii, Topological Methods in the Theory of Nonlinear Integral Equations, Pergamon Press, New York, 1964.

[9] N. S. Papageorgious, On the measurable selection approach in random differential inclusion, fixed point theory and optimizations, J. Math. Phy. Sci. 24 (1990), 331-345.

[10] C. P. Tsokos and W. J. Padgett, Random Integral Equations with Applications to Life Sciences and Engineering, Academic Press, 1974.

"Kasubai", Gurukul Colony, Ahmedpur-413515, Dist: Latur, Maharashtra, India.

E-mail: bcd20012001@yahoo.com 